\title{
Efeito de um protocolo de treinamen- to concorrente sobre fatores de risco para o acúmulo de gordura hepática de adolescentes obesos
}

\section{Effect of a concurrent training on risk factors for the accumulation of hepatic fat of obese adolescents}

Paula A. Monteiro ${ }^{1 *}$, Barbara M. M. Antunes², Loreana S. Silveira1 ${ }^{1}$ Romulo A. Fernandes ${ }^{3}$, Ismael F. Freitas $\mathrm{Jr}^{3}$

\begin{abstract}
RESUMO
Modelo de estudo: Estudo prospectivo

Objetivo: Avaliar o efeito de um protocolo de treinamento concorrente com duração de 16 semanas sobre fatores de risco para o acúmulo de gordura hepática de jovens obesos. Metodologia: A amostra foi formada por 38 indivíduos obesos de ambos os sexos e com idade entre 12 e 15 anos. A obesidade foi atestada pelo percentual de gordura corporal, o qual foi estimado pela absortometria radiológica de dupla energia (DEXA). Adicionalmente, a quantidade de gordura localizada no tronco $(\mathrm{kg})$ foi estimada também. Antes e após a intervenção, os jovens foram submetidos a exames bioquímicos de sangue (perfil lipídico completo em jejum [mg/dL]) e a ultrassonografia do fígado (tamanho dos lobos direito [LD $\mathrm{em} \mathrm{cm}$ ] e esquerdo [LE em cm]). A Intervenção consistiu de treinamento concorrente (treino resistido [30 minutos] e aeróbio [30 minutos]) com três sessões semanais, totalizando 180 minutos por semana. A análise estatística foi composta pelo teste $t$ de Student para dados pareados, utilizando o software SPSS (17.0), e significância estatística fixada em $p<5 \%$. Resultados: Após a intervenção, foram observadas melhoras significantes no percentual de gordura total (PRÉ: $45,1 \pm 5,3$ e PÓs: $41,7 \pm 5,6$; $p=0,001$ ) e na região do tronco (PRÉ: 46,5 $\pm 5,6$ e PÓs: $42,9 \pm 6,3 ; p=0,001$ ). Para o perfil lipídico, houve redução no colesterol total (PRÉ: $164 \pm 34$ e PÓs: $148 \pm 29$; $p=0,001$ ), triglicérides (PRÉ: $118 \pm 59$ e PÓs: $104 \pm 53$; $p=$ 0,002 ) e lipoproteínas de baixa densidade (PRÉ: $100 \pm 29$ e PÓS: $85 \pm 26 ; p=0,001$ ), porém, não para as de alta densidade $(p=0,981)$. Tanto o LE (PRÉ: $8,8 \pm 1,4$ e PÓs: $7,8 \pm 1,3 ; p=0,001)$ como o LD (PRÉ: 13,6 $\pm 1,3$ e PÓs: $12,9 \pm 1,1 ; p=0,001)$ sofreram diminuição em suas proporções. Conclusão: Em jovens obesos, o treinamento concorrente foi eficiente no combate a alguns fatores de risco ao acúmulo de gordura no fígado, bem como, na redução da gordura em ambos os lobos do órgão.
\end{abstract}

Palavras chave: Treinamento. Obesidade. Fígado Gorduroso.

1- Departamento de Fisioterapia - Universidade Estadual Paulista, Campus de Presidente Prudente, São Paulo, Brasil.

2- Departamento de Educação Física - Universidade Estadual Paulista, Campus de Rio Claro, São Paulo, Brasil.

3- Departamento de Educação Física - Universidade Estadual Paulista, Campus de Presidente Prudente, São Paulo, Brasil.
Correspondência:

Paula Alves Monteiro

Rua Redentore Gonfiantine, 160. Vila São Francisco. São

Paulo - São Paulo.

CEP 05351020. Tel 551137669493. email: paulinha_1003@ hotmail.com 


\section{Introdução}

O aumento da prevalência da obesidade associa-se ao desenvolvimento de doenças cardiovasculares e metabólicas, ${ }^{1}$ bem como a doença gordurosa não alcoólica do fígado (DGNAF), a qual é influenciada por alterações no perfil lipídico, resistência à insulina e acúmulo excessivo de gordura visceral. ${ }^{2}$ Atualmente estudos indicam a DGNAF como uma variável de risco para o desenvolvimento da síndrome metabólica (SM), ${ }^{3,4}$ por ser uma doença fortemente associada com resistência à insulina, dislipidemia, obesidade e diabetes mellitus tipo 24.

A DGNAF é comumente caracterizada pelo acúmulo de gordura nos hepatócitos, o qual pode evoluir até mesmo ao câncer hepático. ${ }^{5} \mathrm{~A}$ prevalência da DGNAF em crianças magras é de aproximadamente $2,5 \%$ e pode alcançar $52 \%$ em jovens obesos. ${ }^{6} \mathrm{~A}$ forma de tratamento sugerida para a DGNAF em indivíduos obesos é a diminuição do excesso de gordura corporal por meio de controle alimentar e prática regular e sistematizada de exercícios físicos. ${ }^{7,8}$ Tal procedimento contribui para o tratamento da DGNAF, bem como a outras disfunções associadas. ${ }^{9}$

Em função dos efeitos benefícios da prática de exercícios na prevenção e tratamento da DGNAF, alguns modelos de treinamento, estão sendo estudados. ${ }^{8,10}$ Em relação ao tratamento da obesidade por meio da prática de exercícios físicos, os modelos de treinamento mais utilizados são aqueles que incluem atividades aeróbias. Estudos evidenciam que este tipo de treinamento está fortemente associado ao aumento do gasto energético e ativação da lipólise,${ }^{11}$ melhoria do quadro de resistência à insulina e perfil lipídico alterado. ${ }^{12}$ Outro modelo de treino é o resistido, ou treinamento com peso, que mesmo não propiciando tantas alterações no peso corporal, tem sido utilizado por seu efeito na composição corporal, como o ganho de massa corporal magra (MCM) o qual, por sua vez, pode auxiliar na redução dos fatores de risco associados à obesidade. ${ }^{13}$

A associação de exercícios aeróbios e resistidos, denominado de "treino concorrente" (TC), pode propiciar resultados mais efetivos do que a realização isolada de um dos modelos. ${ }^{14,15} \mathrm{O}$ potencial desse modelo de treino já tem sido evidenciado tanto em adultos, ${ }^{16,17}$ como em crianças e adolescentes que apresentam obesidade, perfil lipídico alterado e síndrome metabólica. ${ }^{14,18}$ Contudo, ainda é pouco investigado o efeito em adolescentes obesos e seus indicadores de gordura hepática. Diante do exposto, o obje- tivo do presente estudo foi avaliar o efeito de um protocolo de treinamento concorrente com duração de 16 semanas sobre fatores de risco para o acúmulo de gordura hepática de jovens obesos.

\section{Métodos}

O presente estudo, de caráter longitudinal, realizou-se no Departamento de Educação Física da Universidade Estadual Paulista - UNESP câmpus de Presidente Prudente, no ano de 2010 e atendeu crianças e adolescentes da cidade e região. Para participar do presente estudo, adotaram-se os seguintes critérios de inclusão: (a) o jovem ser classificado como obeso pelo índice de Massa Corporal (IMC) destinado a população pediátrica, de acordo com idade e faixa etária, seguindo critério publicado por Cole et al 19; (b) ter entre 12 e 15 anos completos na data da avaliação; (c) não apresentar nenhum problema de ordem clínica que impossibilite a prática de atividades físicas e; (d) os pais ou responsáveis legais assinarem o termo de consentimento formal para participação no programa. Os participantes que atingiram três faltas consecutivas ou aleatórias durante a intervenção foram excluídos do referente programa de extensão

Foram avaliados 60 jovens, 22 deles foram excluídos da amostra por excesso de faltas (presença mínima de $95 \%$ no programa). Desta forma, para a presente pesquisa fizeram parte da amostra 38 adolescentes obesos, participantes do programa para tratamento da obesidade. Todos os participantes passaram por exame médico antes de iniciarem as atividades, ocasião na qual foi feito o encaminhamento para a realização dos exames bioquímicos de sangue e ultrassonografia. Ao final do estudo, os exames médicos foram realizados novamente.

Do ponto de vista ético, todos os cuidados foram tomados. Cada sujeito foi convidado a participar, voluntariamente, do estudo e, juntamente com seus pais ou responsáveis legais, informados detalhadamente sobre objetivos e, também, como os dados seriam coletados. Somente participaram aqueles que apresentaram o termo de consentimento formal e esclarecido, devidamente assinado. O presente estudo foi aprovado pelo Comitê de Ética em Pesquisa da FCT/ UNESP com número de protocolo 07/2009.

\section{Triagem e avaliações iniciais}

A primeira triagem foi composta de mensuração da massa corporal e da estatura, para classifica- 
ção do estado nutricional, por meio do IMC, seguindo os valores de referência de Cole et al. ${ }^{19}$ Em seguida realizaram exame bioquímico de sangue ultrassonografia e foram submetidos à avaliação da composição corporal pela densitometria óssea. Todas essas avaliações foram repetidas ao final do estudo.

\section{Avaliação da composição corporal e análises bioquímicas do sangue}

Para a análise da composição corporal e da distribuição da gordura corporal foi empregada a técnica da absorptiometria de raios- $X$ de dupla energia (Dualenergy $\mathrm{x}$-ray absorptiometry), utilizando-se o equipamento modelo GE Lunar - DPX-NT. Desta forma foram estimadas o percentual de gordura corporal (\%GC) e do tronco (GT).

Para as análises bioquímicas foram respeitados um jejum de 12 horas. A coleta foi realizada em tubo a vácuo com gel separador sem anticoagulante; após a coleta, o sangue foi centrifugado por $10 \mathrm{minu}-$ tos a $3.000 \mathrm{rpm}$ para separar o soro dos demais componentes do sangue, sendo que o soro é que vai ser utilizado para as análises. Para dosagem da glicemia, triglicérides, colesterol total e suas frações (HDLColesterol, LDL-Colesterol), foi utilizado um kit enzimático colorimétrico processado em um aparelho Autohumalyzer A5. ${ }^{20}$

\section{Ultrassonografia}

O exame de ultrassonografia de abdômen superior foi realizado, seguindo as recomendações médicas e literárias, com jejum de 4 horas, para verificar tamanho do lobo direito (LD) e esquerdo (LE) do fígado. O tamanho do fígado foi medido do diâmetro crânio-caudal da porção posterior do fígado, através de uma linha oblíqua traçada entre a extremidade superior e a borda hepática inferior. O exame foi realizado sempre pelo mesmo médico radiologista e utilizando o mesmo equipamento (marca TOSHIBA, modelo Aplio).

\section{Treinamento concorrente}

Durante 16 semanas, o programa de treinamento concorrente (TC) foi conduzido com frequência semanal de três vezes e duração de 60 minutos por sessão (aproximadamente 50\% do tempo do treino aeróbio e $50 \%$ do tempo do treino resistido [musculação]). ${ }^{21}$ Foram realizados exercícios de alongamento no início e final do treino e os participantes foram orientados a ingerir água e trajar roupas adequadas para a prática de exercícios físicos.

Para a determinação da intensidade do treino aeróbio, os adolescentes realizaram um teste de esforço máximo progressivo e continuo em esteira ergométrica modelo ATL (marca Inbrasport, BR), para usuários de até $180 \mathrm{~kg}$, com 0 a $26 \%$ de inclinação e velocidade de até $24 \mathrm{~km} / \mathrm{h}$. O teste de esforço iniciou-se a uma velocidade de $3 \mathrm{~km} / \mathrm{h}$, durante um minuto a uma inclinação de $1 \%$. A cada um minuto a velocidade foi aumentada em $0,5 \mathrm{~km} / \mathrm{h}$ mantendo-se a inclinação em $1 \%$, respeitando um período máximo de 12 minutos de teste. ${ }^{22}$ Durante todo o teste de esforço os avaliados permaneceram com uma máscara de silicone com pneumotacografo de médio fluxo (10 até 120 1/min). Por meio do teste de esforço estabelecer-se-á o $\mathrm{VO}_{2}$ pico e a frequência cardíaca deste momento para o controle da intensidade do treinamento.

O treino aeróbio consistiu de caminhada e corrida em uma pista de atletismo oficial de 400m de extensão. A intensidade do esforço foi monitorada por meio do monitor de frequência cardíaca (marca Polar® S810) colocado, aleatoriamente em quatro indivíduos a cada sessão. O equipamento foi ajustado para que se tivesse garantia de que os participantes permanecessem na zona aeróbia de treino (1-4 semana: $65 \% \mathrm{VO}_{2}$ pico; 5-8 semana: $70 \% \mathrm{VO}_{2}$ pico; 9-12 semana: $80 \% \mathrm{VO}_{2}$ pico; $13-16$ semana: $85 \% \mathrm{VO}_{2}$ pico), para isso foi utilizada a frequência cardíaca encontrada na mesma intensidade durante a realização do teste de esforço máximo.

Para, a determinação da Intensidade do Treinamento Resistido, após o período de 4 semanas de adaptação, os avaliados foram submetidos ao teste de predição de uma carga máxima por meio do teste de 10 repetições máximas (10RM), segundo Baechle, ${ }^{23}$ com uma carga máxima individual para a realização de 10 repetições em cada exercício. Para o teste de 10 RM realizou-se os exercícios Leg Press $45^{\circ}$, Supino reto, Remada baixa e Rosca direta, a fim de analisar grandes grupos musculares. Após o período de adaptação o treino foi iniciado com intensidade de $40 \%$ e acrescido de $5 \%$ a cada duas semanas, totalizando uma intensidade final de 55\% (Quadro I).

\section{Análise Estatística}

A estatística descritiva foi composta por valores de média e desvio-padrão. As comparações ao longo do tempo foram efetuadas pelo teste $t$ de Student para dados pareados, utilizando o software SPSS (17.0) e significância estatística fixada em $\mathrm{p}<5 \%$. 
Quadro I

Intensidade do treino resistido.

\begin{tabular}{|c|c|c|c|c|}
\hline & $N^{o}$ de semanas & Intensidade & $N^{o}$ série & $N^{o}$ repetições \\
\hline \multirow[t]{2}{*}{ Etapa 1} & 2 & Adaptação & 1 & 20 \\
\hline & 2 & (cargas mínimas) & 1 & 25 \\
\hline \multirow[t]{2}{*}{ Etapa 2} & 2 & $40 \% \mathrm{RM}$ & 1 & 20 \\
\hline & 2 & $40 \% \mathrm{RM}$ & 1 & 20 \\
\hline \multirow[t]{2}{*}{ Etapa 3} & 2 & $45 \% \mathrm{RM}$ & 1 & 20 \\
\hline & 2 & $45 \% \mathrm{RM}$ & 1 & 20 \\
\hline \multirow[t]{2}{*}{ Etapa 4} & 2 & $50 \% \mathrm{RM}$ & 1 & 15 \\
\hline & 2 & $50 \% \mathrm{RM}$ & 1 & 15 \\
\hline \multirow[t]{2}{*}{ Etapa 5} & 2 & $55 \% \mathrm{RM}$ & 1 & 15 \\
\hline & 2 & $55 \% \mathrm{RM}$ & 1 & 15 \\
\hline
\end{tabular}

\section{Resultados}

Na Tabela 1 são apresentados os resultados das características gerais da composição corporal, nos momentos pré-treinamento e pós-treinamento. Foi possível observar que o treinamento físico resultou em reduções significativas no peso corporal $(p=0,001)$, $\operatorname{IMC}(\mathrm{p}=0,001), \% \mathrm{GC}(\mathrm{p}=0,001)$ e na GT $(\mathrm{p}=0,001)$.

A Figura 1 mostra as variáveis bioquímicas analisadas, sendo que foi possível verificar que o treina-

\section{Tabela 1}

Parâmetros ponderais de adolescentes obesos, pré e pós dezesseis semanas de treinamento concorrente.

\begin{tabular}{lcc}
\hline & $\begin{array}{c}\text { Pré } \\
\text { Média } \pm \boldsymbol{D P}\end{array}$ & $\begin{array}{c}\text { Pós } \\
\text { Média } \pm \boldsymbol{D P}\end{array}$ \\
\hline Peso $(\mathbf{k g})$ & $83,2 \pm 16,5$ & $81,0 \pm 15,5$ \\
Estatura $(\mathbf{c m})$ & $161,9 \pm 8,2$ & $163,4 \pm 8,2$ \\
IMC & $31,5 \pm 4,7$ & $30,1 \pm 4,4$ \\
\%GC & $45,1 \pm 5,3$ & $41,7 \pm 5,6$ \\
\%GT & $46,5 \pm 5,6$ & $42,9 \pm 6,3$ \\
\hline
\end{tabular}

IMC= Índice de Massa Corporal; \%GC= Percentual de Massa Gorda; $\% G T=$ Percentual de Gordura na região do Tronco. mento propiciou a diminuição nos níveis de triglicérides $(\mathrm{p}=0,002)$, colesterol total ( $\mathrm{p}=0,001)$ e LDL-Colesterol $(\mathrm{p}=0,001)$, já o nível do HDL-Colesterol não houve alteração após dezesseis semanas de treinamento concorrente $(\mathrm{p}=0,981)$.

A Figura 2 mostra o efeito do treinamento concorrente após dezesseis semanas sobre as dimensões dos lobos hepáticos, direito e esquerdo, sendo possível verificar diminuição significante das dimensões tanto do lobo direito $(\mathrm{p}=0,002)$ como do esquerdo $(\mathrm{p}=0,001)$.

\section{Discussão}

O acúmulo excessivo de lipídios em forma de tecido adiposo, principalmente na região visceral, e dislipidemia são tidos como variáveis de risco para a DGNAF. ${ }^{24} \mathrm{O}$ exercício físico pode ser considerado uma ferramenta importante no tratamento e na prevenção da obesidade, bem como da DGNAF, ${ }^{8}$ da mesma forma que a inatividade física está relacionada com dislipidemia e pode contribuir, parcialmente, para o risco de doenças como a aterosclerose, diabetes mellitus, obesidade e a DGNAF. ${ }^{25,26}$

Os benefícios do treinamento concorrente foram evidenciados quando analisamos os dados do presente estudo, nos quais as variáveis relacionadas com a composição corporal, perfil lipídico (exceto HDL-C) e dimensões do fígado apresentaram diminuição signi- 


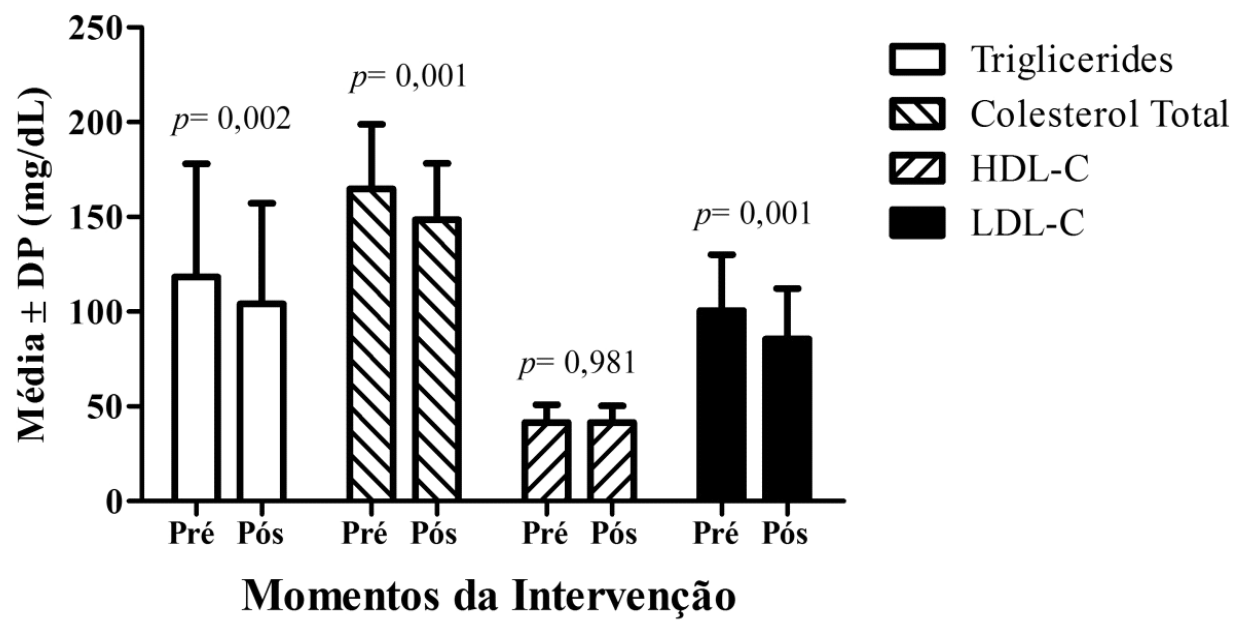

Figura 1. Variáveis bioquímicas em adolescentes obesos, pré e pós dezesseis semanas de treinamento concorrente.

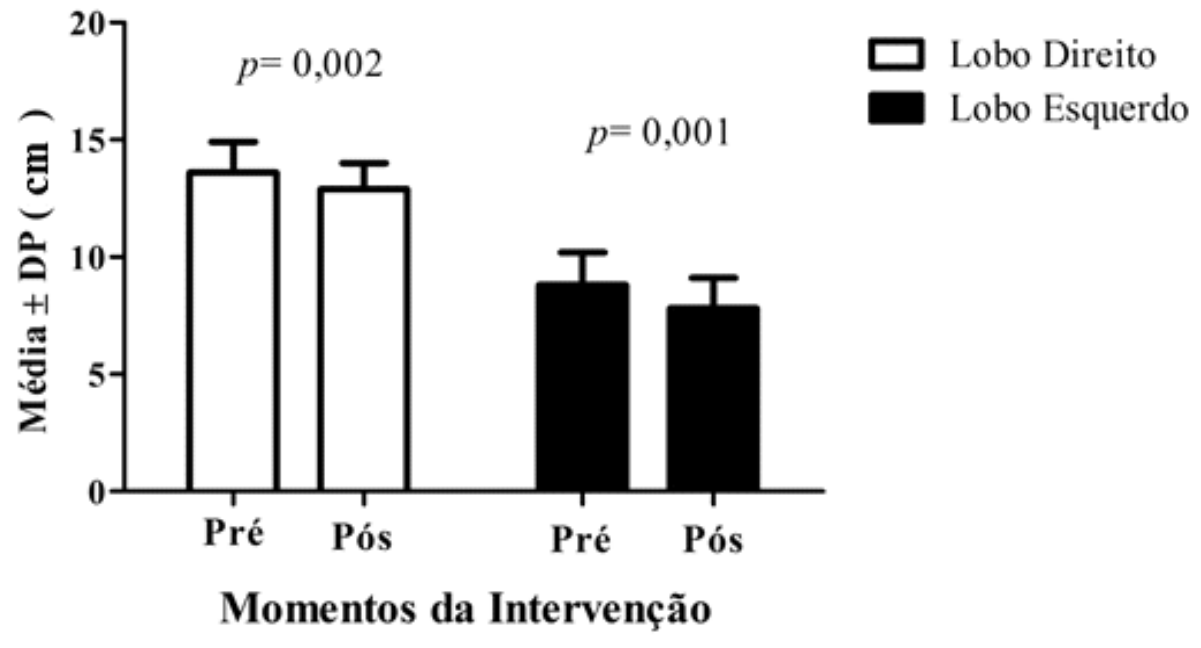

Figura 2. Dimensões dos lobos hepáticos direito e esquerdo em adolescentes obesos, pré e pós dezesseis semanas de treinamento concorrente

ficante. Keating et al., ${ }^{8}$ relatam que o exercício físico por si só é capaz de diminuir o acúmulo de gordura no fígado independente das alterações na composição corporal, já Davis et al. ${ }^{14}$ em sua pesquisa realizada com adolescentes com excesso de peso, relata também haver efeito do treinamento concorrente em medidas de adiposidade e não para variáveis metabólicas.

No presente estudo, dentre as variáveis lipídicas, a única variável que não apresentou resposta significante ao treinamento foi o HDL-C. Possivelmente, está ausência de resultado pode ter sido influenciada pela intensidade do exercício adotado no protocolo, pois, estudos anteriores ${ }^{27}$ indicam que as alterações nas frações de HDL-C parecem ser mais afetadas pelas atividades de alta intensidade. Em nosso estudo, por se tratar de jovens sedentários e com excesso de peso, a prática de atividades física foi mantida apenas em intensidades moderadas e não trabalhou-se intensidade mais elevadas. Por outro lado, informações anteriores oferecem suporte à afirmação de que jovens obesos devem ser estimulados à prática de exercício fisico de maior intensidade objetivando a prevenção de lesão endotelial derivado de dislipidemia. ${ }^{28}$ Assim, sugere que futuros estudos analisem o desenvolvimento de modelos de exercícios em intensidade mais elevadas. 
O tamanho do órgão hepático pode também relacionar-se com a presença da DGNAF, tendo em vista que o acúmulo excessivo de gordura pode alterar suas dimensões29. A hepatomegalia, caracterizada pelo aumento das dimensões do fígado, em função do excesso de lípides neste órgão, em casos agudos da DGNAF, podem desencadear em dores e incomodo abdominal30. Neste sentido, a diminuição das dimensões do figado na presente pesquisa mostra-se efetiva, como ferramenta de controle no diagnóstico da DGNAF em adolescentes obesos31. A mobilização e subsequente utilização da gordura hepática durante e após o exercício parece ser ferramenta importante no que se refere à indicação deste tipo de exercício físico na prevenção e tratamento da DGNAF entre jovens obesos.

A ausência de grupo controle deve ser indicada como limitação do estudo, bem como, indicação para futuros estudos. De fato, manter jovens obesos em comportamento sedentário (grupo controle) pode receber críticas por questões éticas, assim, a utilização de modelos de experimento em "cross-over" parece ser uma indicação adequada.

Diante dos resultados obtidos é possivel concluir que em jovens obesos, o treinamento concorrente pode ser eficiente no combate à dislipidemia e acúmulo de gordura no fígado, demonstrada pela redução no tamanho de ambos os lobos do órgão.

\begin{abstract}
Study Design: Prospective study

Objective: To evaluate the effect of a protocol of concurrent training lasting 16 weeks on risk factors for the accumulation of hepatic fat in obese youth. Methods: 38 obese children and adolescents of both sexes, between 12 and 15 years old. The obesity was attested by the percentage of body fat, which was estimated by dual-energy X-ray absorptiometry (DEXA). Additionally, the amount of fat located in the trunk $(\mathrm{kg})$ was estimated too. Before and after the intervention, the youths underwent biochemical blood tests (fasting complete lipid profile [mg / dL]) and ultrasonography of the liver (right size Wolves [LD cm] and left [LE in $\mathrm{cm}]$ ). The intervention consisted of concurrent training (strength training [30 minutes] and endurance training [30 minutes]) with three sessions per week, totaling 180 minutes a week, for ten weeks. Statistical analysis was made by the test $t$ of Student for paired data using SPSS software (17.0) and significance statistical fixed at $p<5 \%$. Results: After the intervention, significant improvements were observed in the percentage of total fat (PRE: $45.1 \pm 5.3$ and POST: $41.7 \pm 5.6, p=0.001$ ) and in the trunk region (PRE: $46,5 \pm 5.6$ and POST: $42.9 \pm 6.3, p=0.001$ ). For lipid profile, reduction in total cholesterol (PRE: $164 \pm 34$ and POST: $148 \pm 29, p=0.001$ ), triglycerides (PRE: $118 \pm 59$ and POST: $104 \pm 53, p=$ 0.002 ) and lipoproteins density (PRE: $100 \pm 29$ and POST: $85 \pm 26, p=0.001$ ), but not for high-density ( $p$ $=0.981$ ). Both the LE (PRE: $8.8 \pm 1.4$ and POST: $7.8 \pm 1.3, p=0.001$ ) and LD (PRE: $13.6 \pm 1.3$ and POST: $12.9 \pm 1,1, p=0.001$ ) experienced a decrease in its proportions. Conclusion: The concurrent training was effective in combating some risk factors to the accumulation of fat in the liver, as well as in reducing fat in both lobes of the organ in young obese.
\end{abstract}

Keywords: Training. Obesity. Fatty Liver.

\section{Referências}

1. Lavrador MSF, Abbes PT; Schimith MAM. Escrivão, José Augusto de Aguiar, Carrazedo Taddei. Riscos Cardiovasculares em Adolescentes com Diferentes Graus de Obesidade. Arq Bras Cardiol.. 2011; 96: 205-11.

2. Bhatia LS, Curzen NP, Calder PC, Byrne CD. Non-alcoholic fatty liver disease: a new and important cardiovascular risk factor? Eur Heart J. 2012; 33: 1190-200.

3. Chaves GV, Souza DS, Pereira SE, Saboya CJ, Peres WAF. Associação entre doença hepática gordurosa não alcoólica e marcadores de lesão/função hepática com componentes da síndrome metabólica em indivíduos obesos classe III. Rev Assoc Med Bras. 2012; 58: 288-93.
4. El-Koofy NM, Anwar GM, El-Raziky MS, El-Hennawy AM, ElMougy FM, El-Karaksy HM, et al. . The association of metabolic syndrome, insulin resistance and non-alcoholic fatty liver disease in overweight/obese children. Saudi J Gastroenterol. 2012; 18: 44-9.

5. Neuman G., Sagi R., Shalitin S., Shimon R. Serum Inflammatory Markers in Overweight Children and Adolescents with NonAlcoholic Fatty Liver Disease. ISR Med Assoc J. 2010; 12 : 410-5.

6. Araujo MB. Doença hepática gordurosa não alcoólica e obesidade. Rev Abeso 2010; 43: 43.

7. Johnson NA, George J. Fitness versus fatness: moving beyond weight loss in Anonalcoholic fatty liver disease. Hepatology. 2010; 52: 370-81. 
8. Keating SE, Hackett DA, George J, Johnson NA. Exercise and non-alcoholic fatty liver disease: A systematic review and meta-analysis. J hepatol. 2012; 57: 157-66.

9. Padilha PC, Rocha HF, Alves N, Peres WAF. Prevalência de doença hepática não-alcoólica em crianças e adolescentes obesos: uma revisão sistemática. Rev Paul Pediatr. 2010; 28: 387-93.

10. Guttierres APM, Marins JCB. Os efeitos do treinamento de força sobre os fatores de risco da síndrome metabólica. Rev Bras Epidemiol. 2008; 11: 147-58.

11. Chaudhary S, Kang MK, Sandhu JS. The Effects of Aerobic Versus Resistance Training on Cardiovascular Fitness in Obese Sedentary Females. Asian, J Sports Med. 2010; 1: 177-84.

12. Guo W, Kawano H, Piao L, Itoh N, Node K, Sato T. Effects of Aerobic Exercise on Lipid Profiles and High Molecular Weight Adiponectin in Japanese Workers. Intern Med. 2011; 50: 38995.

13. Strasser B, Arvandi M, Siebert U. Resistance training, visceral obesity and inflammatory response: a review of the evidence. Obesity. 2012; 8: 1-14.

14. Davis JN, Tung A, Chak SS, Ventura EE, Byrd-Williams CE, Alexander KE, et al. Aerobic and Strength Training Reduces Adiposity in Overweight Latina Adolescents. Med Sci Sports Exerc. 2009; 41: 1494-503.

15. Donnelly JE, Blair SN, Jakicic JM, Manore MM, Rankin JW, Smith BK. American College of Sports Medicine Position Stand. Appropriate physical activity intervention strategies for weight loss and prevention of weight regain for adults. Med Sci Sports Exerc. 2009; 41: 459-71.

16. Libardi CA, Souza GV, Gáspari AF, Dos Santos CF, Leite ST, Dias $R$, et al. Effects of concurrent training on interleukin-6, tumour necrosis factor-alpha and C-reactive protein in middle-aged men. Med Sci Sports Exerc. 2011; 29: 1573-81.

17. Silva RF, Cadore EL, Kothe G, Guedes M, Alberton CL, Pinto SS. Concurrent training with different aerobic exercises. Int J Sports Med. 2012; 33: 627-34.

18. Mello MT, Piano A, Carnier J, Sanches Pde L, Corrêa FA, Tock L. et al. Long-Term Effects of aerobic plus resistance training on the metabolic syndrome and adponectinemia in obese adolescents. J Clin Hypertens. 2011; 13: 343-50.
19. Cole TJ, Bellizzi MC, Flegal KM, Dietz William.Establishing a standard definition for child overweight and obesity worldwide: international survey. Br Med J. 2000; 320: 1240-3.

20. Human RP, Jones GA. Evaluation of swab transport systems against a published standard. J Clin Pathol. 2004; 57: 762-3.

21. Faigenbaum AD, Westcoyy WL. Youth strength training: programs for health. Fitness and sport. 2nd. Ed. Human Kinetcs; 2009.

22. Bentley DJ, Newell J, Bishop D. Incremental exercise test design and analysis implications for performance diagnostics in endurance athletes. Sports Med. 2007; 37: 575-86.

23. Baechle TR, Groves BR, Weigth Training. Chapaign: Leissure Press.1992.

24. Finelli C, Tarantino G. Is Visceral Fat Reduction Necessary to Favour Metabolic Changes in the Liver? J Gastrointest Liver Dis.. 2012 Jun; 21: 205-8.

25. Lira FS, Rosa JC, Lima-Silva AE, Souza HA, Caperuto EC Seelaender MC, et al. Sedentary subjects have higher PAI-1 and lipoproteins levels than highly trained athletes. Diabetol Metab Syndr. 2010; 2: 7.

26. Magkos F, Lavoie JM, Kantartzis K, Gastaldelli A. Diet and exercise in the treatment of Fatty liver. J Nutr Metab. 2012; 2012: 2.

27. Kraus WE, Houmard JA, Duscha BD, Knetzger KJ, Wharton MB, McCartney JS, et al. Effects of the amount and intensity of exercise on plasma lipoproteins. N Engl J Med. 2002; 347: 1483-92.

28. Wong VWS. Recent advances in the management of nonalcoholic fatty liver disease. Med Bull. 2008; 13:19-22.

29. Reis KAA, Roquete MLV, Penna FJ. Esteatohepatite nãoalcoólica na infância e adolescência: revisão. Pediatria (São Paulo). 2001; 23: 329-39.

30. Roberts EA. Pediatric nonalcoholic fatty liver disease (NAFLD) A "growing" problem? J Hepatol. 2007; 46: 1133-42.

31. Damaso AR, Tock L, Tufik S, Prado WL, Stella SG, Fisberg M. Tratamento multidisciplinar reduz o tecido adipose visceral, leptina, grelina e a prevelência de esteatose hepática não alcoólica (NAFLD) em adolescentes obesos. Rev Bras Med Esporte. 2006; 12: 263-7. 